\title{
The modeling design integral the electrostatic microelectromechanical switch of capacitive type
}

\author{
Igor E. Lysenko, Alexey $V$. Tkachenko*, Olga A. Ezhova, and Boris G. Konoplev \\ Southern Federal University, 347929, Taganrog, Russia
}

\begin{abstract}
This article presents the optimization and static modeling of the design an integral microelectromechanical switch of capacitive type based on a parametric 3D model using finite element analysis software complex ANSYS. The values of the pull-down voltage and switching time are determined. The obtained results give a good theoretical help in the development of various high-performance electrostatic microelectromechanical switches.
\end{abstract}

\section{Introduction}

Microelectromechanical systems (MEMS) have achieved tremendous success since their introduction in the 1960s in the form of resonant gate transistors. The development of MEMS technology has led to the emergence of two new classes of devices - sensors and actuators, which continue to replace their macro-scale counterparts due to their miniature size, low cost, low power consumption and possible integration with semiconductor integrated circuit (IC) technology. However, radio-frequency (RF) electronic equipment has always needed switches that make low losses in the open state and provide high isolation in the closed state, with high permissible signal power and low control power.

The first switch to fully meet the requirements of steel electromagnetic relays. After many years of improvement, modern RF electromagnetic relays provide low on signal loss and high isolation in the off state, but they have such disadvantages as large size, high cost and limited life (from hundreds of thousands to tens of millions). Switches on PIN-diodes and field-effect transistors (FET) based on GaAs have a number of advantages over electromagnetic relays, which are high speed operation, small size and weight, as well as low power control. But they are characterized by higher insertion losses and for this reason in a number of applications cannot replace electromechanical switches.

Further search for developers led to the development of RF switches based on MEMS technologies that combined some of the advantages of semiconductor and relay devices. The first reports of MEMS switches developed specifically for RF applications date back to the early 1990s [1-8]. Recently, several micromechanical switches have been manufactured using MEMS technologies.

\footnotetext{
*Corresponding author: msqk@mail.ru
} 
The transition to micro-scale RF MEMS switches is reflected in the significant growth of the market of RF MEMS segment and their applications. Typical applications of RF MEMS switches are tele- and radio communications, industry, mobile devices, base stations, radars, antenna switches. According to the marketing research and analysis of technologies conducted by Yole Developpment Group, the average annual growth rate of profit of the RF segment of MEMS market is $10 \%$ per year: from 2,3 billion dollars in 2018 to 4,4 billion dollars in 2024, which exceeds the average annual growth rate of profit from such segments of MEMS market as: inertial sensors, pressure sensors, microphones and optical MEMS devices [9]. This expansion of the RF MEMS segment is due to the successful transition of these technological solutions from the research the commercial sector. In the commercial sector, these devices often experience uncertain and frequently changing environmental conditions that can significantly affect their performance and reliability.

In this article we carried out modeling and optimization of the developed design of the integral MEMS switch of capacitive type by means of methods of the finite element analysis in the ANSYS software complex.

\section{Description of the design of a MEMS switch}

MEMS switches can be classified into three main parameters: (1) the structure or type of inclusion in the RF electrical circuit - serial or parallel inclusion; (2) the type of design of the mechanical switching element - cantilever or cantilever and membrane; (3) the type of contact - resistive (metal-metal) and capacitive (metal-dielectric-metal). This article presents a study of the design of the membrane MEMS switch with capacitive contact type in the RF electrical circuit developed by the authors. The main elements of the switch design are shown in Fig. 1.

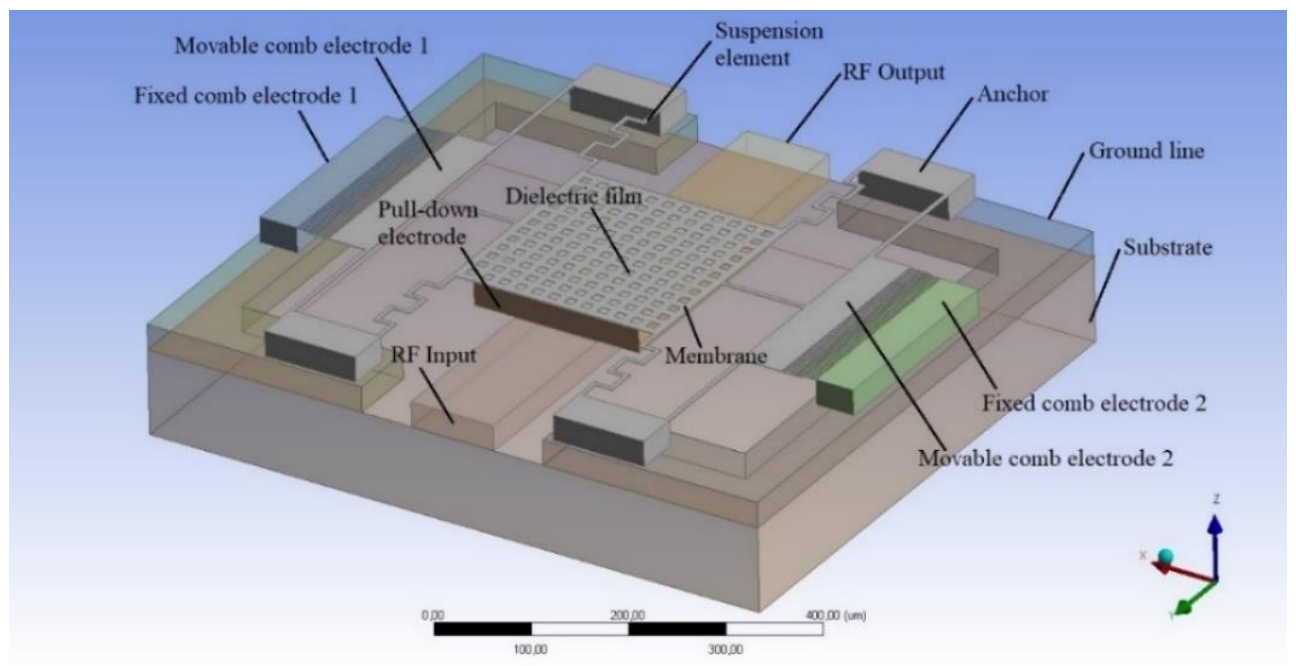

Fig. 1. Design and main elements of MEMS switch.

As shown in Fig. 1 the switch design uses a perforated metal membrane as the switching mechanism. The membrane is fixed to the anchor areas by means of elastic suspension elements with an air gap relative to the RF transmission line of the coplanar waveguide, as well as relative to the fixed pull-down electrode. A dielectric film is applied to the surface of the fixed pull-down electrode. Thus, the RF transmission line forms a 
variable capacitance capacitor with the grounded membrane. At the same time, in the case of the down position of the membrane (in contact with the dielectric film of the membrane), the RF signal entering the input line is shunted to the grounding lines of the coplanar waveguide.

In addition, the design of the presented switch provides an electrostatic clamp, which is formed by two comb drives: (1) movable comb electrode 1 - fixed comb electrode 1; (2) movable comb electrode 2 - fixed comb electrode 2 . This electrostatic clamp is designed to hold the membrane in its original position (in the case when the switch is open and the RF signal with low losses enters the RF output of the transmission line) when its structure is affected by linear accelerations, shock and vibration induced by shock pulses. This is because linear accelerations, mechanical shocks and vibrations are the three main environmental influences that can cause unwanted short circuits or open RF transmission lines. In turn, this will cause errors and malfunction of the entire RF device or the entire RF system into which the switch is integrated.

In addition to the influence of environmental factors to malfunction MEMS switch leads Brownian noise. Brownian noise leads to a random change in capacitance, which in turn leads to additional phase and amplitude noise at the RF output of the device. In this regard, the quality indicator $Q$ of a well-designed device should lie within: $0,3 \leq Q \leq 3$ so that the switching time is limited only by the mechanical parameters (mass, stiffness coefficient, etc.) of the switch design.

The quality index of MEMS structure is determined by the Equation (1):

$$
Q=\left(\frac{k}{\omega_{0} b}\right)
$$

where $k$ - the stiffness coefficient; $\omega_{0}=\sqrt{\frac{k}{m}}$ - the angular frequency; $m-$ the mass of the structure; $b$ - the damping coefficient.

The geometric parameters of the structural elements of the MEMS switch are given in Table 1 .

Table 1. Geometric parameters of MEMS switch design elements

\begin{tabular}{|c|c|}
\hline Structural element & $\begin{array}{c}\text { Dimensions (length } \mathrm{x} \text { width } \mathrm{x} \\
\text { thickness), } \mu \mathrm{m}\end{array}$ \\
\hline Membrane & $250 \times 250 \times 1$ \\
\hline Fixed pull-down electrode & $245 \times 200 \times 10$ \\
\hline Dielectric film & $245 \times 200 \times 0,15$ \\
\hline Suspension element (meander) & $35 \times 5 \times 1$ \\
\hline Anchor & $110 \times 50 \times 30$ \\
\hline Hole in membrane & $10 \times 10 \times 1$ \\
\hline
\end{tabular}

The materials of the main structural elements of MEMS switch is aluminum [10], and as the dielectric material is used $\mathrm{Si}_{3} N_{4}$. 


\section{Theoretical basis}

Since the MEMS switch designs have small deviations at the micro level, their mechanical behavior can be analyzed by knowing the stiffness constant of the elastic suspension elements $k(\mathrm{~N} / \mathrm{m})$ [11]. In the design of the proposed switch elastic suspension elements have a serpentine shape.

Thus, the stiffness constant is calculated by determining the stiffness coefficient of individual elements, as shown in Fig. 2, followed by finding the effective stiffness coefficient $k_{\text {eff }}$ [12]. The Equation (2) for determining the effective stiffness coefficient $k$ has the form:

$$
k=E w\left(\frac{t}{l}\right)^{3}
$$

where $E$-Young's modulus; $w$-the width; $t$ - the thickness; $l$ - the length of the beam.

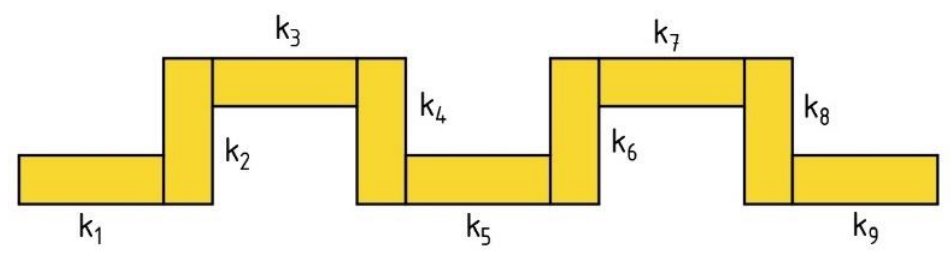

Fig. 2. An elastic suspension element having two square meanders grouped from individual elements with constant stiffness $k$.

Thus, the average value of the constant stiffness of the elastic element $k_{\text {eff }}$, shown in Fig. 2, defined by Equation (3):

$$
\frac{1}{k_{m}}=\frac{1}{k_{1}}+\frac{1}{k_{2}}+\frac{1}{k_{3}}+\frac{1}{k_{4}}+\frac{1}{k_{5}}+\frac{1}{k_{6}}+\frac{1}{k_{7}}+\frac{1}{k_{8}}+\frac{1}{k_{9}}
$$

The effective stiffness constant $k_{\text {eff }}$ can be determined by Equation (4):

$$
k_{\text {eff }}=4 k_{m}
$$

MEMS switches are triggered when an electrostatic force is present between the fixed pull-down electrode and the membrane. This force causes the membrane to deflect and come into contact with the RF transmission line [11]. The magnitude of the pull-down voltage is determined by the Equation (5):

$$
V_{p}=V\left(\frac{2}{3}\right)=g_{0}=\sqrt{\frac{8 k g_{0}^{3}}{27 \varepsilon_{0} W w}}
$$

where $g_{0}$ - the air gap value; $\varepsilon_{0}$ - the vacuum permittivity; $W$ - the width of the membrane; $w$ - the width of the fixed pull-down electrode.

The switching time $t_{s}$ is used to determine the switching speed. This is the rate at which the design of the switching element - membrane passes from one state to another. The mechanical regenerative switching force is increased by the use of higher voltages [12]. The switching time depends on the magnitude of the pull-down voltage $V_{p}$ and the supply voltage $V_{s}=1.4 V_{p}$ and is approximately determined by the Equation (5): 


$$
t_{s} \approx \frac{3.67 V_{p}}{V_{s}}
$$

The capacitance ratio is one of the key parameters of capacitive MEMS switches and is defined as the ratio of the capacitance of the variable capacitor in the down position of the membrane and in the initial position, which are given by Equation (6) and Equation (7):

$$
\begin{gathered}
C_{u}=\frac{\varepsilon_{0} A}{g_{0}+\frac{t_{d}}{\varepsilon_{r}}} \\
C_{d}=\frac{\varepsilon_{0} \varepsilon_{r} A}{t_{d}}
\end{gathered}
$$

where $t_{d}$-the thickness of the dielectric film; $\varepsilon_{r}$ - the dielectric constant of the material.

In the initial position, the value of the air gap between the membrane and the dielectric film is maximum, and in the down position they are in contact with each other. The capacity ratio is given by the Equation (8):

$$
C_{r}=\frac{C_{d}}{C_{u}}
$$

Resistance MEMS switch consists of two variables $R_{s 1}$ and $R_{s}$, where $R_{s 1}$ due to the loss of RF signal transmission lines of the coplanar waveguide and is defined by Equation (9):

$$
R_{s 1}=2 \alpha Z_{0} l
$$

where $\alpha$ - the RF signal losses in the transmission line of the coplanar waveguide expressed in $\mathrm{Np} / \mathrm{m}$.

The second component of $R_{s}$ is determined by the resistance of the mobile electrode. If the thickness of the movable electrode is less than $2 \delta$, where $\delta$ the depth of the skin layer, the resistance of the switch is constant and does not change with the RF signal frequency. However, if the thickness of the movable electrode is greater than $2 \delta$, the resistivity of the conductor depends on the frequency of the RF signal according to $\sqrt{f}$ in practice, as a rule, the thickness of the movable electrode and the RF MEMS switch design elements are taken equal to $3-4 \delta$. The depth of the skin layer is determined by the Equation (10):

$$
\delta=\sqrt{\frac{2}{\omega \mu \sigma}}
$$

where $\omega=2 \pi f$ - the circular frequency of variable current; $f$ - the resonant frequency; $\mu=4 \pi \cdot 10^{-7}-$ the magnetic constant.

On the basis of the given theoretical data analytical modeling of the presented design of the presented capacitive MEMS switch is carried out [13].

\section{Simulation of mechanical behavior of the device}

The ANSYS program, based on finite element problem solving, provides two different methods for solving electrostatic-structural problems $[14,15]$. This allows you to combine the results of modeling one area to solve related problems. In the case of an Electromechanical problem, the electrostatic and mechanical structural domains are solved 
sequentially. The connection between the two regions is realized by the load vector, which is an electrostatic force acting on the boundary of the structural elements. In ANSYS, each domain is described by a database file called physics environment. In Electromechanical analysis, the interaction between the electrostatic medium and the structural medium is carried out by passing between two relevant databases. The electrostatic problem is based on the solution of the Laplace equation in various homogeneous domains. By solving this equation, it is possible to determine the potential distribution in the environment and on the surface of the conductors and thus calculate the electrostatic force between the two conductors. The next step is solved the structural problem in a structural environment. In fact, the deformed shape of the membrane under the action of electrostatic force is obtained in accordance with the theory of plates/shells [16-18]. The structural region is then switched back to the electrostatic medium, updating the electrostatic grid. The iterative process continues until convergence between the two environments is achieved. The second method of realization of electrostatic-structural problem in ANSYS environment is the method of concentrated or induced order. This method is usually strongly coupled and is considered the fastest in terms of convergence time [19, 20]. It can be implemented using concentrated transducer elements, eliminating the disadvantage of a sequential process. Transducer elements store electrostatic energy and convert it into mechanical energy and conversely. The input data for the transducer elements are the voltage supplied to the opposite nodes, the maximum and minimum air gap. The displacement-capacitance ratio can be obtained from the solution of the electrostatic field problem. Compared to the sequential 3D connection method, electrostatic elements representing the environment (air between two conductors) are replaced by a set of transducer elements. In this way, a simulation of the coupled electrostatic structure can be performed.

In addition, using the second method of solving problems in the ANSYS software package, MEMS switch optimization for specific output parameters can be carried out, as shown in Fig. 3.

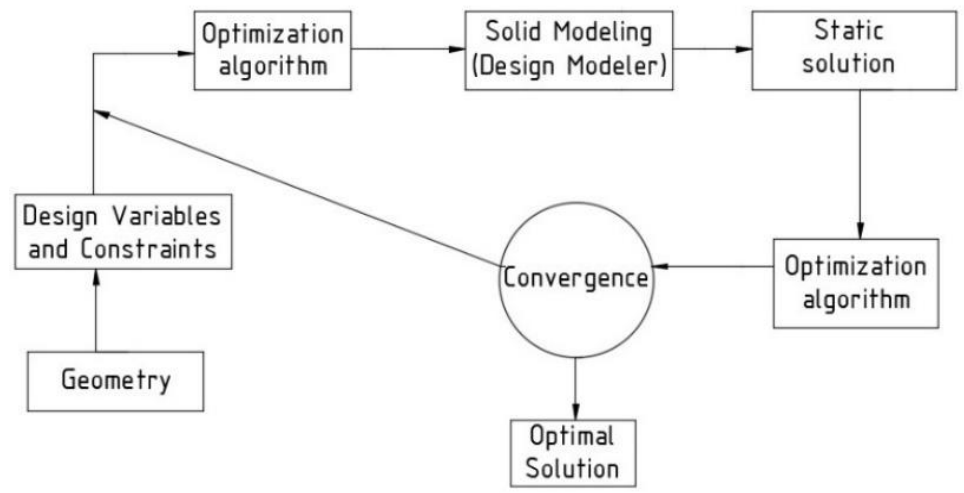

Fig. 3. Traditional approach to MEMS device optimization.

For finite element analysis, the first step is to develop a parametric model of the 3D model of the MEMS switch, which allows you to describe the design of the device using a different set of dimensional characteristics that determine the geometry of the object, the relationship conditions and the relationship of individual elements together.

The parametric 3D model of the switch developed by MEMS is shown in Fig. 4. Parametric 3D model allows you to quickly change one or more dimensions of the structure, which significantly reduces the time for calculations. Each build element is assigned its own unique parameter name, and then the parameter is assigned a numeric value. If you change the value of this parameter, the $3 \mathrm{D}$ model of the switch is automatically recalculated. 

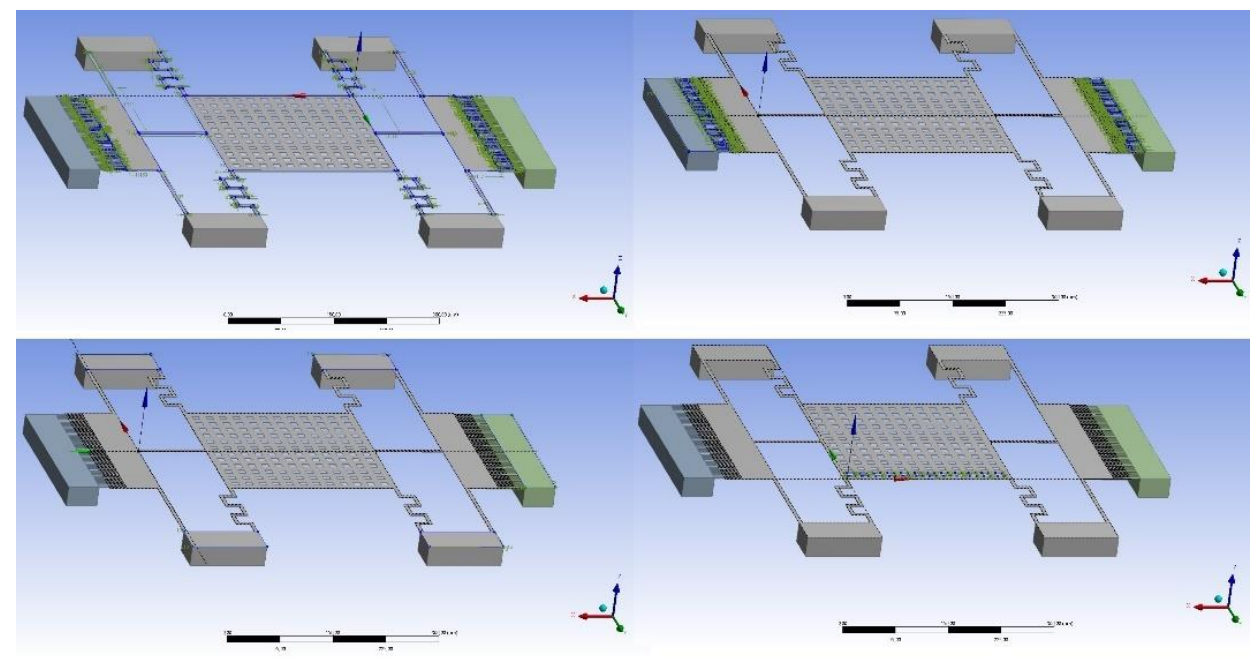

Fig. 4. Parametric 3D model of MEMS switch.

The second important step in numerical simulation of continuum mechanics problems is the quality of the computational finite element mesh. The question of mesh quality is of critical importance in contact problems, since, on the one hand, small elements with higher accuracy reflect the behavior of the structure when loads are applied to it, and on the other hand, such problems, due to their nonlinearity, require significant computational resources. A fragment of a finite element grid with a given characteristic cell size of $15 \mu \mathrm{m}$ is shown in Fig. 5.

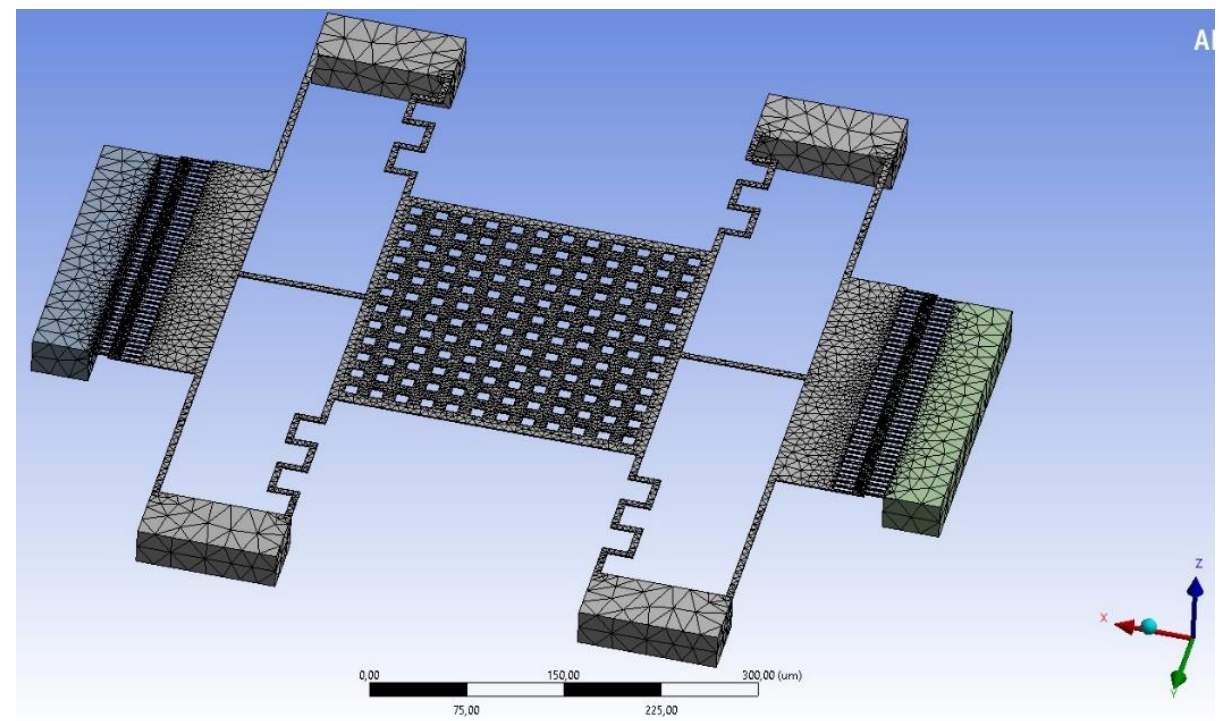

Fig. 5. Finite element meshes parametric 3D model MEMS switch.

When solving a static strength problem in the Static Structural analysis module, displacements, stresses and strains due to applied loads are determined. It is assumed that the effects associated with inertia and damping are small. In Static Structural analysis calculations, user-defined loads are applied incrementally: the solution is broken down into steps. In the first step, displacements and stresses are calculated when a load is applied to the source system that is approximately equal to the partial of the user-defined load divided 
by the number of steps. In the next step, the load is applied to the deformed system, the state of which was determined in the previous step, and so on.

The most acceptable from the point of view of the accuracy of the result and the cost of machine time should be considered a computational experiment performed on a grid with a characteristic cell size of $15 \mu \mathrm{m}$. Fig. 6 illustrates the deformation of the actuator membrane MEMS switch in the down position.

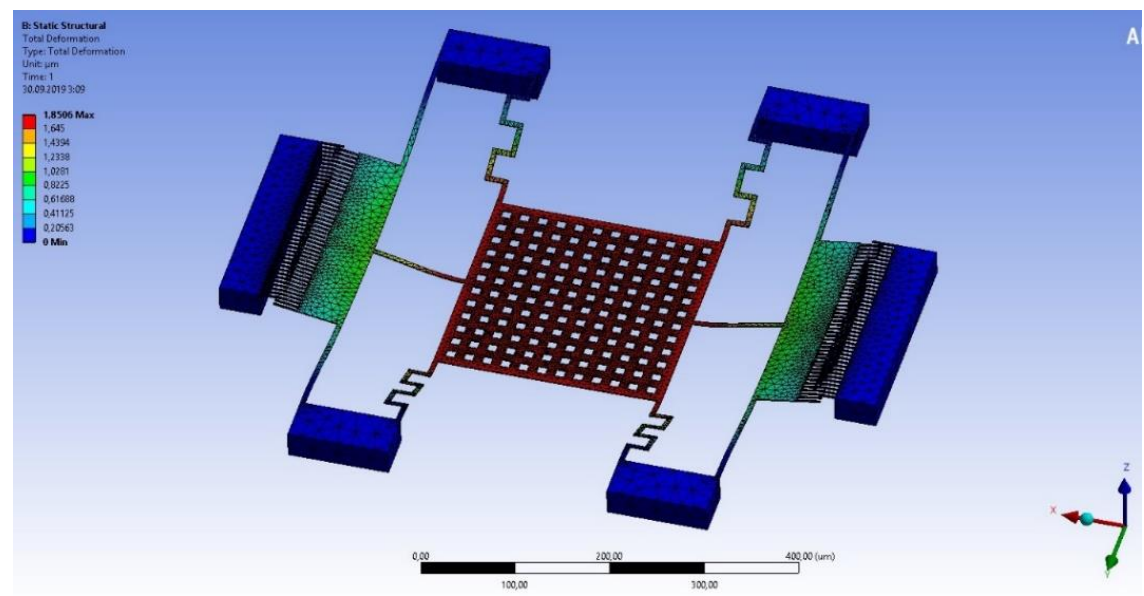

Fig. 6. Deformation of the MEMS switch membrane when an electrostatic field is applied.

For Fig. 7 the dependence of the MEMS switch membrane displacement on time is shown. In this case, the time of switching the membrane to the down position is $5.29 \mu \mathrm{s}$ with the magnitude of the pull-down voltage of $15 \mathrm{~V}$.

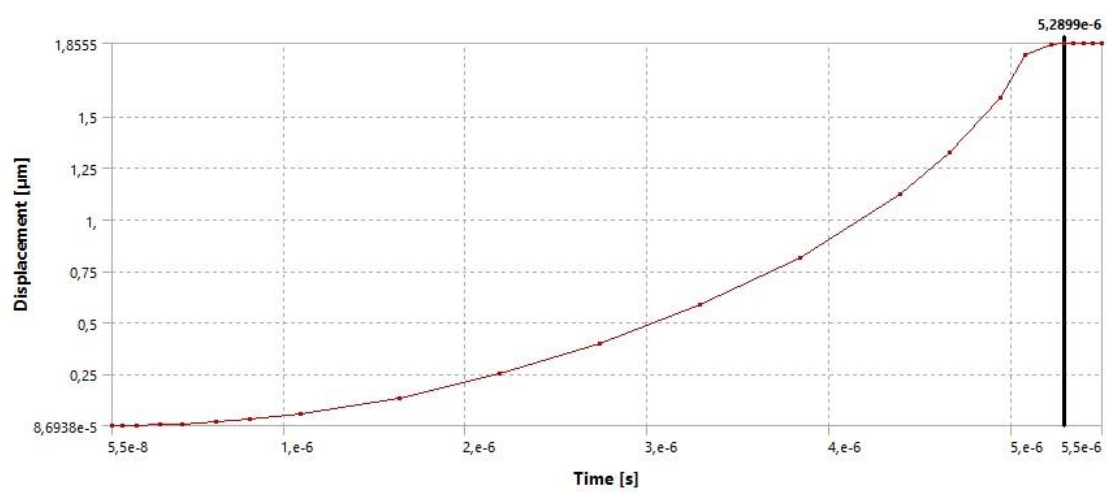

Fig. 7. The dependence of the MEMS membrane displacement of the switch on time.

\section{Conclusion}

In this article, using the ANSYS software package, a parametric 3D model of the capacitive MEMS switch is developed. The optimization of the design using the built-in modules optimization of the design in the ANSYS software package, as well as static finite element modeling using numerical methods to estimate the value of the control voltage and switching time. According to the results of static modeling, the required pull-down voltage for the presented switch design with one central fixed pull-down electrode is $15 \mathrm{~V}$ and the switching time to the down position is $5.29 \mu \mathrm{s}$. The theoretical data presented in this article are the basis for the design of electrostatic MEMS switches. 
The results were obtained using the equipment of the student design bureau "Elements and devices of inertial navigation systems of robotics" of the Institute of Nanotechnologies, Electronics and Electronic Equipment Engineering, Southern Federal University (Taganrog, Russia). This research is supported by the "Program for the Development of the Southern Federal University until 2021" (project VnGr-07/2017-10).

\section{References}

1. L. E. Larson, R. H. Hackett, M. A. Melendes, R. F. Lohr, Micromachined microwave actuator (MIMAC) technology - a new tuning approach for microwave integrated circuits, IEEE 1991 Microwave and Millimeter-Wave Monolithic Circuits Symposium, pp. 27-30 (1991)

2. H. Hosaka, H. Kuwano, K. Yanagiswa, Electromagnetic microrelays: concepts and fundamental characteristics, Sensors and Actuators A, 40, pp. $41-47$ (1994).

3. S. Roy, M. Mehregany, Fabrication of electrostatic nickel microrelays by nickel surface micromachining, Proceedings IEEE Micro Electro Mechanical Systems, pp. 353-357 (1995)

4. J. Simon, S. Saffer, C.-J. Kim, A micromechanical relay with a thermally-driven mercury micro-drop, Proceedings of Ninth International Workshop on Micro Electromechanical Systems, pp. 515-520 (1996)

5. S. Saffer, J. Simon, C.-J. Kim, K. H. Park, J.-H. Lee, Mercury-contact switching with gap-closing microcantilever, Micromachined Devices and Components II, 2882, pp. 204-209 (1996)

6. J. Simon, S. Saffer, F. Sherman, C.-J. Kim, Lateral polysilicon microrelays with a mercury microdrop contact, IEEE Transactions on Industrial Electronics, 45, pp. 854860 (1998)

7. W. P. Taylor, M. G. Allen, Integrated magnetic microrelays: normally open, normally closed, and multi-pole devices, Proceedings of International Solid-State Sensors and Actuators Conference (Transducers'97), pp. 1149-1152 (1997)

8. H. A. C. Tilmans, E. Fullin, H. Ziad, M. D. J. Van de Peer, J. Kesters, E. Van Geffen, J. Bergqvist, M. Pantus, E. Beyne, K. Baert, F. Naso, A fully-packaged electromagnetic microrelay, Technical Digest, IEEE International MEMS'99 Conference, Twelfth IEEE International Conference on Micro Electro Mechanical Systems, pp. 25-30 (1999)

9. D. A. Czaplewski, C. D. Nordquist, G. A. Patrizi, G. M. Kraus, W. D. Cowan, $R F$ MEMS switches with RuO2-Au contacts cycled to 10 Billion cycles, Journal of Microelectromechanical Systems, 22, pp. 655-661 (2013)

10. I. E. Lysenko, A. V. Tkachenko, O. A. Ezhova, Research of the microelectromechanical switch with different materials of metal membrane, Proceeding of SPIE, Proceeding of the International Conference Micro- and Nanoelectronics - 2018 (ICMNE-2018), 10226, pp 1-12 (2019)

11. U. S. Arathy, R. Resmi, Analysis of pull-in voltage of MEMS switches based on material properties and structural parameters, In International Conference on Control, Instrumentation, Communication and Computational Technologies (ICCICCT), pp. 57$61(2015)$

12. K. Srinivasa Rao, T. Lakshmi Narayana, K. Girija Sravani, Design and analysis of CPW based shunt capacitive RF MEMS switch, Cogent Eng, 4, pp. 1-9 (2017)

13. I. E. Lysenko, A. V. Tkachenko, E. V. Sherova, A. V. Nikitin, Analytical approach in the development of RF MEMS switches, Electronics (Switzerland), 7(12), p. 1-23, (2018)

14. J. Taye, K. Guha, S. Baishya, Design and analysis of RF MEMS shunt capacitive switch for low actuation voltage \& high capacitance ratio, In Physics of Semiconductor Devices, pp. 445-448 (2014) 
15. M. Gyimesi, D. Ostergaard, Electro-Mechanical Transducer for MEMS Analysis in ANSYS, Int. Conf. Modeling/Simulation of Microsystems (MSM99), 7, pp. 270-273 (1999)

16. Y. Zhu, H. D. Espinosa, Electromechanical Modeling and Simulation of RF MEMS Switches, 4th International Symposium on MEMS and Nanotechnology, 190, pp. 8-11 (2003)

17. S. Timoshenko, S. Woinowsky-Kreiger, Theory of Plates and Shells, McGraw-Hill, New York (1959)

18. E. Ventsel, T. Krauthamme, Thin Plates and Shells: Theory, Analysis, \& Applications, 1st ed. New York: Marcel Dekker, Inc. (2001)

19. P. Osterberg, H. Yie, X. Cai, J. White, S. Senturia, Self-Consistent Simulation and Modeling of Electrostatically Deformed Diaphragms, 17th IEEE Int. Conference on Microelectromech. Syst., pp. 28-32 (1994)

20. I. V. Avdeev, New Formulation for Finite Element Modeling Electrostatically Driven Microelectromechanical Systems, Ph.D. thesis, University of Pittsburgh, School of Engineering (2003)

21. V. Rochus, Finite element modeling of strong electro-mechanical coupling in MEMS, Ph.D. thesis, University de Liege (2006) 